\title{
Zeolite A Synthesis under Dynamic Conditions, after Hydrogel Ageing
}

\author{
Ana Palčić, ${ }^{a}$ Lavoslav Sekovanić, ${ }^{b}$ Boris Subotić, ${ }^{a}$ and Josip Bronićc ${ }^{a, *}$ \\ ${ }^{a}$ Ruđer Bošković Institute, Bijenička 54, 10000 Zagreb, Croatia \\ ${ }^{\mathrm{b}}$ Geotechnical Faculty, University of Zagreb, Hallerova aleja 7, 42000 Varaždin, Croatia
}

RECEIVED JANUARY 26, 2012; REVISED JULY 6, 2012; ACCEPTED JULY 9, 2012

\begin{abstract}
Zeolites are crystalline, microporous, alumo-silicate materials (around 400 types) which are used in many applications. Each field of usage (catalysis, ion exchange, sorption, molecular sieving, etc.) has demands regarding crystals' structural and particulate properties. Detailed understanding of the mechanisms of crystals' formation and growth enables the control of these properties. Hydrogels of the starting composition $3.2 \mathrm{Na}_{2} \mathrm{O} \times 2 \mathrm{SiO}_{2} \times 1.05 \mathrm{Al}_{2} \mathrm{O}_{3} \times 250 \mathrm{H}_{2} \mathrm{O}$ were heated in stainless steel reactor at $80{ }^{\circ} \mathrm{C}$ under dynamic conditions, after ageing for $t_{\mathrm{A}}=0-209 \mathrm{~h}$ at $25^{\circ} \mathrm{C}$. Solid phase samples were characterized using microscopy (OM, SEM), PXRD and laser light scattering (LLS). Specific number of zeolite A crystals was calculated from particle size distributions. It was shown that the some particulate properties: specific number of crystals and crystal size distribution, are changing towards higher number of crystals and wider size distribution while the morphology of the crystals remains the same. Also, the crystal growth rate is higher in the systems which are treated under dynamic conditions. Obtained results were compared with data of systems crystallized under static conditions and commented on the grounds of the autocatalytic nucleation theory. (doi: 10.5562/cca2055)
\end{abstract}

Keywords: zeolite A, autocatalytic nucleation, crystal size distribution, hydrogel ageing

\section{INTRODUCTION}

Zeolites are crystalline, microporous, alumosilicate materials widely used as catalysts, ion exchangers, molecular sieves, adsorbents, etc., ${ }^{1,2}$ Understanding of basic mechanisms (processes) of zeolite crystals' formation and growth, at molecular level, enables the prediction of their properties. In order to use certain material (powder crystals) for specific application, it is important to produce the crystals of desired properties. In other words, one should be able to control product's chemical $(\mathrm{Al} / \mathrm{Si}$ ratio) and phase (zeolite type) composition as well as their particulate properties (particle size distribution, specific number of crystals and their morphology).

Zeolites are usually synthesized by hydrothermal treatment of (alumino)silicate amorphous hydrogel, which was formed by mixing of alkaline silicate and aluminate solutions. Almost all physico-chemical parameters can have influence on the transformation of hydrogel into zeolite crystals. ${ }^{3}$ The most expressive are the alkalinity of the system, the chemical composition of the gel (concentrations of the aluminate, silicate and added species), and the pretreatment of the gel (ageing, seeding, washing, etc.). Higher activity of $\mathrm{OH}^{-}$ions enables higher concentration of reactive species in liq- uid phase (higher solubility of the gel) and consecutively, higher crystallization rate. ${ }^{3-5}$

Hydrogel ageing causes shortening of the reaction time and lowering the end product's size. ${ }^{4,6}$ In this paper, we have studied the ageing of amorphous precursor and the way it affects the zeolite A nucleation process at dynamic conditions and compared the results with the data obtained from the systems in static conditions. ${ }^{7}$ The proposed model of autocatalytic nucleation is the one that describes the basic processes of zeolite crystallization and is applicable to all systems which have hydrothermal transformation of the gel into relatively low soluble crystals without using structure directing agents. It is based on the assumption that potential crystal nuclei are formed within the alumosilicate gel matrix during its precipitation and during ageing time. These nuclei can grow to crystals only when they are in contact with the reactive species from the solution, which are released to the solution during gel's dissolution. ${ }^{2}$

\section{EXPERIMENTAL}

Aluminosilicate hydrogels were prepared by the addition of alkaline sodium silicate solution into alkaline sodium aluminate solution. Si solution was prepared

\footnotetext{
* Author to whom correspondence should be addressed. (E-mail: bronic@irb.hr)
} 
dissolving of an appropriate amount of fumed silica (99.8 wt. \% $\mathrm{SiO}_{2}$; Sigma) and sodium hydroxide (reagent grade, > 98 wt. \% $\mathrm{NaOH}$, Kemika) in demineralised water, while $\mathrm{Al}$ solution was prepared dissolving of anhydrous sodium aluminate (41 wt. $\% \mathrm{Na}_{2} \mathrm{O}, 54$ wt. $\%$ $\mathrm{Al}_{2} \mathrm{O}_{3}$, Riedel-de-Haën) in demineralised water. $\mathrm{Al}$ solution was purified by centrifugation and only clear solution was used for the preparation of hydrogels. Then, the precipitated hydrogels were dispersed (homogenized), aged for given time $\left(t_{\mathrm{A}}=0,66\right.$ and $\left.209 \mathrm{~h}\right)$ under static conditions in thermally equilibrated bath, at $25{ }^{\circ} \mathrm{C}$, marked as $\mathrm{S} 1-\mathrm{S} 3$, respectively), and poured into stainless steel reaction vessel preheated at $80{ }^{\circ} \mathrm{C}$. Dynamic conditions were obtained via stirring with a Teflon-coated magnetic bar (300 rpm). The ageing time $\left(t_{\mathrm{A}}\right)$ is the time between the moment when two solutions were mixed and the moment when the hydrogel was put into the reactor. At predefined crystallization times $\left(t_{\mathrm{C}}\right)$, aliquots of the reaction mixture were taken and centrifuged to stop the crystallization process and separate the solid from the liquid phase. The solid phase was washed until the $\mathrm{pH} \approx 9$, dried at $105^{\circ} \mathrm{C}$ overnight, and used for the characterization.

The phase composition of the samples was determined by powder X-ray diffraction (PXRD) using Philips PW 1820 diffractometer $\left(\mathrm{CuK}_{\alpha}\right.$ radiation, $2 \theta=$ $5-50^{\circ}$ ). The weight fraction of zeolite A in each sample, $f_{\mathrm{C}}$, was calculated on the grounds of the obtained XRD patterns ( 1 diffractogram -1 point). Obtained data represent the crystallization curves when drawn as $f_{\mathrm{C}}$ against time of crystallization, $t_{\mathrm{C}}$. The size of the largest crystals $\left(L_{\mathrm{m}}\right)$ at various crystallization times $\left(t_{\mathrm{C}}\right)$ was determined by the Zhdanov and Samulevich's method [8], using optical (Meji equipped with Nikon Coolpix 990 digital camera) and scanning electron microscope (SEM, Jeol 7000). Crystal (particle) size distribution (CSD) curves were obtained using laser light scattering particle size analyzer (Malvern Mastersizer 2000). Specific number of crystals $\left(N_{\mathrm{S}}\right)$ was calculated from corresponding CSD curves, normalized to unit value of $1 \mathrm{~g}$ of zeolite A:

$$
N_{S}=\frac{1}{G \rho \sum\left(N_{i}\left(D_{i}\right)^{3}\right)}
$$

where $N_{i}$ - the number of the crystals within segment $\left(D_{i} \pm \Delta D / 2\right), D_{i}-$ (average) particle diameter within segment (sphere equivalent diameter), $\rho$ - the density of zeolite $\mathrm{A}\left(2 \mathrm{~g} \mathrm{~cm}^{-3}\right)$, and $G$ - the geometrical shape factor (for sphere $=1$ ). The nucleation curves represent the rate of "nuclei appearance" $\left(\mathrm{d} N / \mathrm{d} t_{\mathrm{C}}\right)$ in the liquid phase of the system within time of crystallisation $\left(\mathrm{d} N / \mathrm{d} t_{\mathrm{C}}\right.$ versus $\left.t_{\mathrm{C}}\right)$. They were calculated according to the Zhdanov and Samulevich's method [8]. From the cumulative nucleation curves, the fractions of "appear-
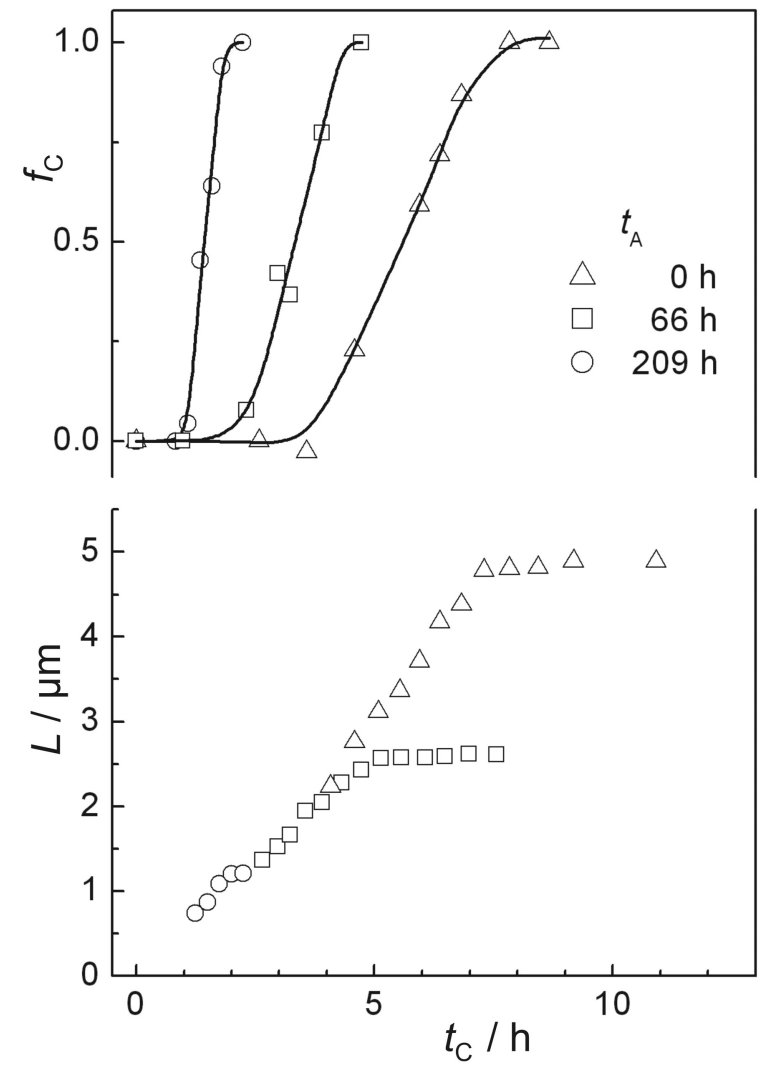

Figure 1. Fraction, $f_{\mathrm{C}}$, of the zeolite A crystallized during transformation of the hydrogel aged $\left(t_{\mathrm{A}}\right)$ for $0 \mathrm{~h}(\triangle), 66 \mathrm{~h}(\square)$ and $209 \mathrm{~h}(\bigcirc)$. Symbols are experimental points (samples), while crystallization curves are drawn for clarity. $L$ - average size of the largest crystals obtained from photos made by optical $(\triangle, \square)$ and scanning electronic $(\bigcirc)$ microscope.

ing nuclei" in the system, $f_{\mathrm{N}}$, was calculated. The curves, which show dependence of the number fraction $\left(f_{\mathrm{N}}\right)$ of nuclei on the amount of crystalline material $\left(f_{\mathrm{C}}\right)$, represent the distributions of the nuclei within gel matrix., ${ }^{2,7}$

\section{RESULTS AND DISCUSSION}

Crystallization curves (Figure 1) show that the kinetics of the gel transformation to zeolite A gets faster with hydrogel ageing time. From the size of largest crystals (Zhdanov-Samulevich's method: ${ }^{8}$ Figure 1) can be seen that the curve for S2 is shifted towards longer crystallization time for ca $0.5 \mathrm{~h}$ (still, it is within experimental error). The size of the largest crystals of the final product (column $L_{\mathrm{m}}$ in Table 1), shows decreasing values with ageing time (crystallization time too) and both are in accordance with previous findings. ${ }^{7,-11}$ Average crystallization rate of the studied systems (using the same method of calculation) is significantly faster $\left(0.684 \mu \mathrm{m} \mathrm{h}^{-1}\right)$ than the crystallization rate of the ones transformed under static conditions $\left(0.618 \mu \mathrm{m} \mathrm{h}^{-1}\right)$. 
Table 1. The numerical values of measured and calculated parameters relevant for the describing of zeolite A crystals formed from aged hydrogels in dynamic conditions: $t_{\mathrm{A}}$-gel ageing time, $L_{\mathrm{m}}$ - the size of the largest crystals determined by ZhdanovSamulevich's method, $D_{(\mathrm{V} 0.5)}$ - value in $\mu \mathrm{m}$ under which $50 \%$ of particles lie in CSD by volume, $D_{(\mathrm{N} 0.5)}-$ value in $\mu \mathrm{m}$ under which $50 \%$ of particles lie in CSD by number, $a_{\mathrm{S}}\left(\mathrm{m}^{2} \mathrm{~g}^{-1}\right)$ - specific surface area of crystals, and $N_{\mathrm{S}}-$ specific number of crystals, both obtained from the particle size distribution by volume.

\begin{tabular}{ccccccc}
\hline System & $t_{\mathrm{A}} / \mathrm{h}$ & $L_{\mathrm{m}} / \mu \mathrm{m}$ & $D_{(\mathrm{V} 0.5)} / \mu \mathrm{m}$ & $D_{(\mathrm{N} 0.5)} / \mu \mathrm{m}$ & $a_{\mathrm{S}} / \mathrm{m}^{2} \mathrm{~g}^{-1}$ & $N_{\mathrm{S}} / \mathrm{g}^{-1}$ \\
\hline $\mathrm{S} 1$ & 0 & 4.89 & 2.832 & 1.286 & 2.46 & $2.053 \times 10^{11}$ \\
$\mathrm{~S} 2$ & 66 & 2.62 & 2.555 & 1.988 & 2.45 & $2.702 \times 10^{11}$ \\
$\mathrm{~S} 3$ & 209 & $1.20^{*}$ & 1.418 & 0.902 & 4.56 & $9.493 \times 10^{11}$ \\
\hline
\end{tabular}

* The $L_{\mathrm{m}}$ value measured from SEM photos, the rest are measured from OM photos.

The SEM photos of the final product obtained by crystallization of hydrogels aged $\left(t_{\mathrm{A}}\right)$ for 0,66 and $209 \mathrm{~h}$ were shown in Figure 2. They confirm that the ageing process affects final product's size producing smaller crystals with increasing ageing time of the starting hydrogels.

Since the diffraction halo of visible light around the particles smaller than $1.5 \mu \mathrm{m}$ gives overestimation of their size, only SEM images of the end products of the hydrogel aged for $209 \mathrm{~h}$ were taken (S3) for the determination of largest crystals. The sizes of the largest crystals, $L_{\mathrm{m}}$, show that the value drops from 4.89 $\mu \mathrm{m}$ obtained from fresh hydrogel to 1.19 from hydrogel aged for $209 \mathrm{~h}$, indicating that the specific number of crystals increase as it is shown in column $N_{\mathrm{S}}$ in Table 1.

Crystal size distributions by volume and number of the final products (Figure 3) show that the particles obtained from fresh hydrogel are the largest (also widest distribution), while the particles obtained from the hydrogel aged for $209 \mathrm{~h}$ are the smallest (also narrowest distribution) which is in good agreement with the $L_{\mathrm{m}}$ measurements. It is important to note that all distributions are monomodal.

Due to the correlation between volume and number size distributions of the same sample, it is normal that $D_{(\mathrm{N} 0.5)}$ value for number distribution is shifted towards lower value than for volume distribution $\left(D_{(\mathrm{V} 0.5)}\right.$, Table 1). A large number of small crystals in systems $\mathrm{S} 3$ and S1 show strong maxima at $0.45-1.0 \mu \mathrm{m}$ and causes significant shifting of $D_{(\mathrm{N} 0.5)}$ value (Figure 3 ). Lower value for $D_{(\mathrm{N} 0.5)}$ of the fresh system $(1.286 \mu \mathrm{m}$, i.e. stronger shifting) than for the system aged for $66 \mathrm{~h}$ $(1.988 \mu \mathrm{m})$ is assigned to the large number of small crystals (below $1 \mu \mathrm{m}$ ) in fresh system.

Data of the specific surface area (column $a_{\mathrm{s}}$ in Table 1) show significant difference between the system aged for $t_{\mathrm{A}}=209 \mathrm{~h}$ and other systems. While $a_{\mathrm{s}}$ has nearly the same value for systems S1 and S2 (2.46$2.45 \mathrm{~m}^{2} \mathrm{~g}^{-1}$ ), in the $\mathrm{S} 3$ system it becomes almost two times higher $\left(4.56 \mathrm{~m}^{2} \mathrm{~g}^{-1}\right)$. This fact is a consequence of much smaller crystals (their higher specific number) than in other systems.
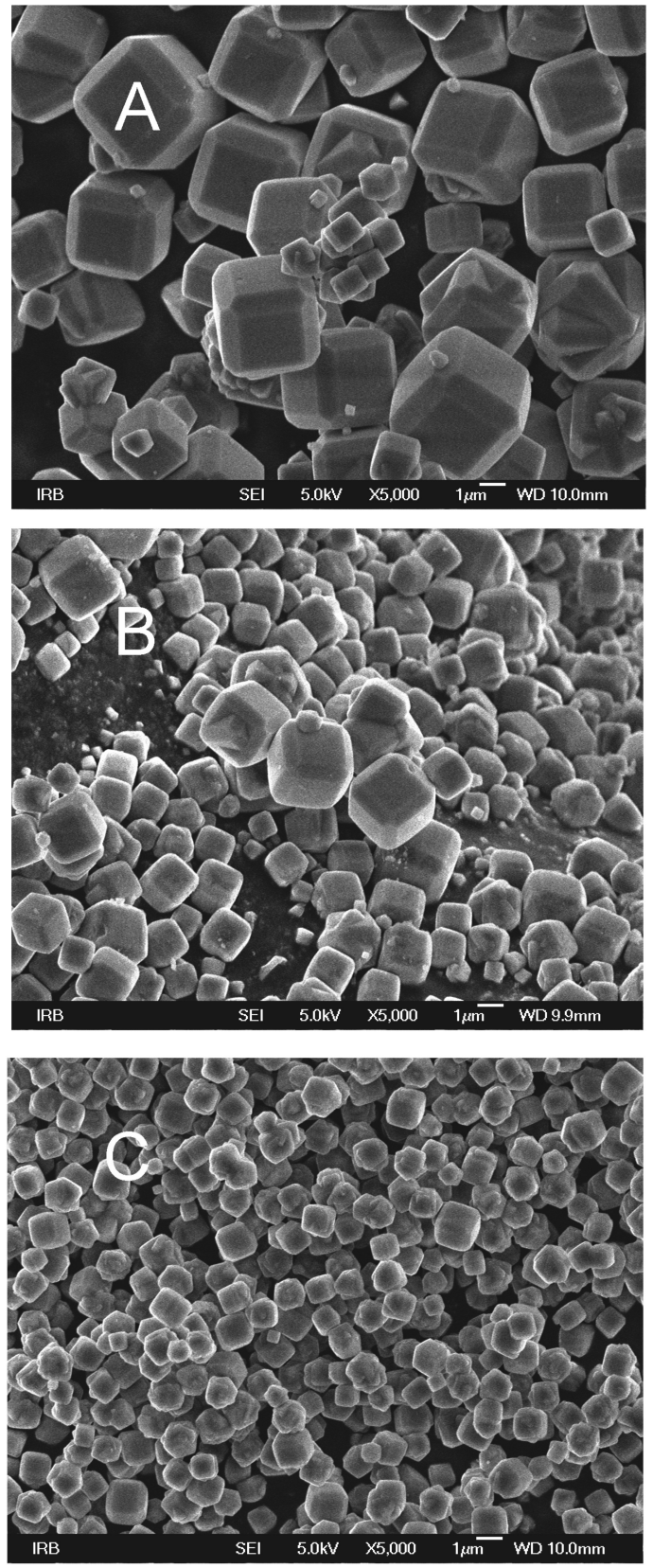

Figure 2. SEM photos of the zeolite A crystals formed after transformation of hydrogels aged $\left(t_{\mathrm{A}}\right)$ for $0 \mathrm{~h}(\mathrm{~A}), 66 \mathrm{~h}(\mathrm{~B})$, and $209 \mathrm{~h}(\mathrm{C})$. 

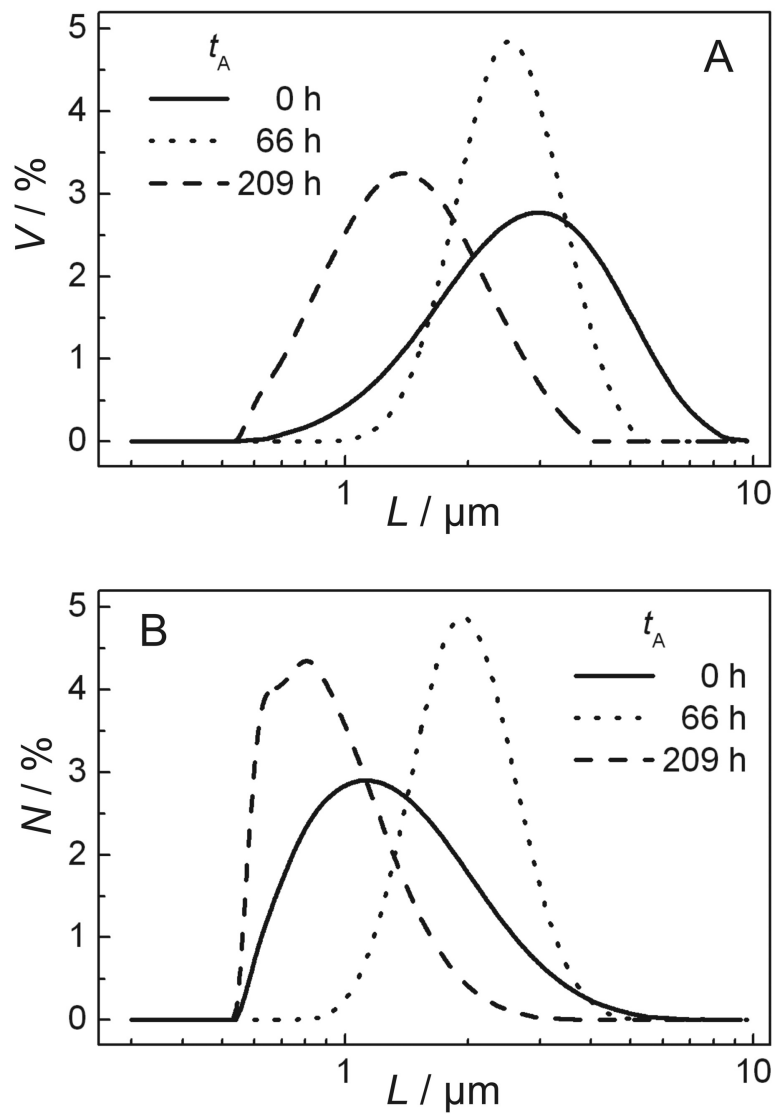

Figure 3. Particle size distribution curves by volume (A) and by number (B) at the end of crystallization of zeolite A aged $\left(t_{\mathrm{A}}\right)$ for $0 \mathrm{~h}$ (full line), $66 \mathrm{~h}$ (dotted line) and $209 \mathrm{~h}$ (dashed line).

Specific number of particles in studied systems is the total number of crystals within $1 \mathrm{~g}$ of the zeolite A, obtained at the end of the transformation. The values calculated from corresponding crystal size distributions are listed in Table 1. Specific number increases with ageing time from $2.053 \times 10^{11}$ to $9.493 \times 10^{11}$. They are somewhat higher than the values for systems crystallized under static conditions. This behavior is expected even with the first idea of the model of autocatalytic crystallization $^{12}$ and is completely in accordance with findings of Cundy et al., on synthesis of silicalite-1 and titanosilicalite- $1 .^{13}$

Curves of the nucleation (maybe better expression is the curves of the nuclei appearance in the system, i.e. the time when they begin to grow) are shown in Figure 4, and used for the calculation of the nuclei distribution within gel matrix. They are similar to the curves of the systems crystallized at static conditions. ${ }^{7}$ The major difference between the systems done under static and dynamic conditions of the synthesis is the time of transformation, and consecutively, different values of kinetics' parameters and distributions of nuclei within gel matrix.

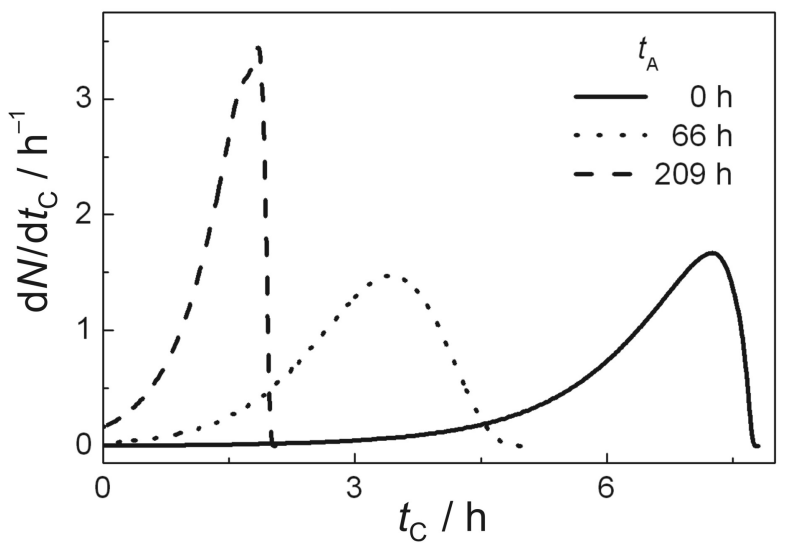

Figure 4. The nucleation curves of the systems S1-S3 aged $\left(t_{\mathrm{A}}\right)$ for $0 \mathrm{~h}$ (full line), $66 \mathrm{~h}$ (dotted line) and $209 \mathrm{~h}$ (dashed line). $\mathrm{d} N / \mathrm{d} t_{\mathrm{C}}-$ the nucleation rate (the number fraction of nuclei), $t_{\mathrm{C}}$ - the time of crystallisation.

The comparison of the distribution of the nuclei within hydrogels (Figure 5) transformed under static (filled symbols) and dynamic conditions (outlined symbols) show that the nuclei are similarly distributed within gel matrix. Ideal homogeneous distribution of the nuclei in gel matrix is straight line between 0 and 1 in $f_{\mathrm{N}}$ $-f_{\mathrm{C}}$ graph, but it was never found in real systems. The beginnings of all curves are similar in the systems aged for the same time - triangles for fresh systems (S1),

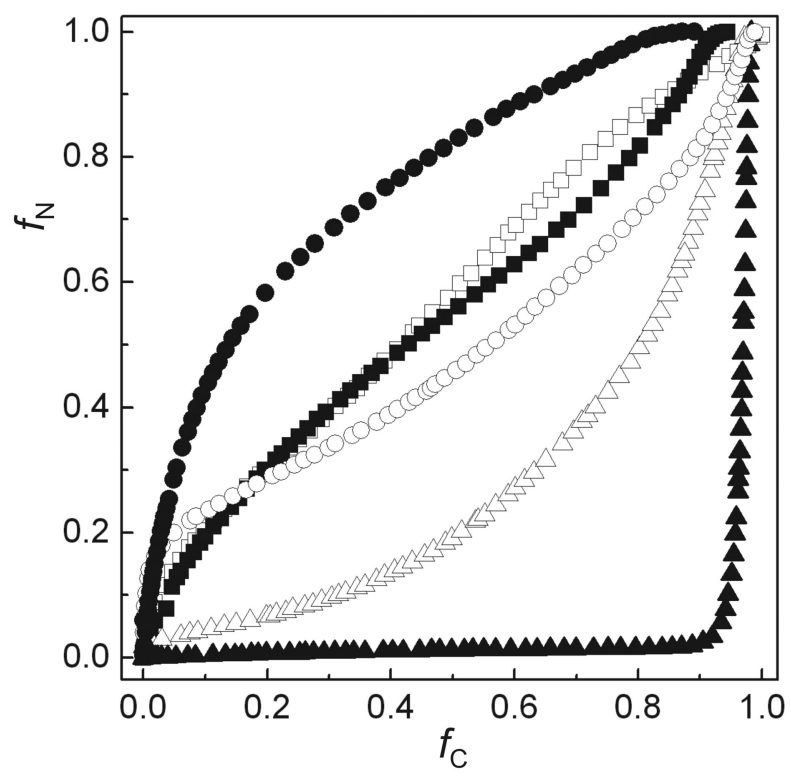

Figure 5. Curves of zeolite A nuclei distribution within gel matrix. Systems were aged for $0 \mathrm{~h}(\triangle), 66 \mathrm{~h}(\square)$ and $209 \mathrm{~h}$ $(\bigcirc)$. Filled symbols are for systems crystallized under static conditions, while outlined symbols stand for systems under dynamic conditions (magnetic stirrer bar, $300 \mathrm{rpm}$ ). $f_{\mathrm{C}}-$ fraction of the zeolite A crystals, $f_{\mathrm{N}}$ - number fraction of nuclei. 
squares for $\mathrm{S} 2$ and circles for $\mathrm{S} 3$. Changes in the systems' behavior arise very fast after the beginning of the reactions, due to the better homogeneity and hydrodynamics of the stirred system. Namely, processes of the crystal growth are controlled by the diffusion of the reactive species (low molecular aluminate, silicate and alumosilicate species) from the place where they are created (dissolution of the gel particles) to the surface of the growing crystals (nuclei). Mobility of the reactive species is much better in stirred systems, and the distribution of the gel and formed crystals within volume of the reaction solution is more homogeneous (shorter time from creation to the deposition of the reactive species). The consequence is faster crystal growth and synergy effect of the quicker gel dissolution.

These results confirm the basic thesis of the autocatalytic nucleation theory: crystal nuclei are dominantly formed during hydrogel precipitation and during hydrogel ageing, due to the reactions of reactive species from solution with the surface and subsurface area of gel particles. Since the nuclei can grow only in contact with solution, they need to be released from gel matrix. An increase of the gel ageing time enables the formation of more nuclei close to the surface (subsurface) of the gel area, which are quickly released to the solution from the gel matrix at the beginning of the crystallization (fast increase of the number of nuclei circles in Figure 5). Therefore, overall reaction is faster, crystals are smaller and their specific number is higher. This is in accordance with the above presented results, since all prepared hydrogels have the same chemical composition, but dynamic synthesis conditions (stirring) accelerate crystallization process. The increase of the gel ageing also raise its transformation rate through generation of more nuclei at gels' (sub)surface area.

\section{CONCLUSION}

The basic theory of the autocatalytic nucleation that crystal nuclei are dominantly formed during hydrogel precipitation and during hydrogel ageing is valid regardless of stirring (dynamic or static conditions) of the reaction hydrogel.
An increase of the gel ageing time enables formation of more nuclei close to the surface (subsurface) of the gel area which is confirmed in both modes of crystallization (static and dynamic conditions).

The changes of the behavior of the systems in dynamic conditions (compared to the static ones) arise very fast after beginning of the reactions, due to the better homogeneity (Figure 5) and hydrodynamics of the stirred system. The consequence is faster crystal growth rate as well as the quicker gel dissolution.

Dynamic conditions (stirring) of the reaction hydrogel accelerate crystallization as well as an increase of the gel ageing time. Therefore, in both cases, overall reaction is faster, crystals are smaller and their specific number is higher.

Acknowledgements. This work was supported by the Ministry of Science, Education and Sport of Republic of Croatia within basic project 098-0982904-2953.

\section{REFERENCES}

1. P. Parya, P. K. Dutta, in: "Handbook of Zeolite Science and Technology", S. M. Auerbach, K. A. Carrado, and P. K. Dutta (Eds.), Marcel Dekker Inc., New York - Basel, 2003, 1-19.

2. B. Subotić, J. Bronić, T. Antonić Jelić, in: Ordered Porous Sol$i d s$, V. Valtchev, S. Mintova, M. Tsapatsis (Eds), Elsevier, Amsterdam, 2008, 127-185.

3. B. Subotić, J. Bronić, in: "Handbook of Zeolite Science and Technology", S.M. Auerbach, K. A. Carrado, and P.K. Dutta (Eds.), Marcel Dekker Inc., New York - Basel, 2003, 129-203.

4. J. Bronić, B. Subotić, I. Šmit, and Lj. A. Despotović, Stud. Surf. Sci. Catal. 37 (1988) 107-114.

5. B. Tokay and A. Erdem-Senatalar, Micropor. Mesopor. Mater. 148 (2012) 43-52.

6. H. Lechert, Stud. Surf. Sci. Catal. 18 (1984) 107-113.

7. A. Palčić, J. Bronić, Đ. Brlek, and B. Subotić, CrystEngComm. 13 (2011) 1215-1220.

8. S. P. Zhdanov and N. N. Samulevich, In: Proc. of the Fifth International Conference on Zeolites, Heyden, L. V. Rees (Ed.), London-Philadelphia-Rheine, 1980, 75-84.

9. P. M. Slangen, J. C. Jansen, and H. van Bekkum, Micropor. Mater. 9 (1997) 259-265.

10. L. Gora and R. W. Thopmson, Zeolites 18 (1997) 132-141.

11. S. Bosnar, J. Bronić, Đ. Brlek, and B. Subotić, Micropor. Mesopor. Mater. 142 (2011) 389-397.

12. S. P. Zhdanov, Adv. Chem. Ser. 101 (1971) 1-24.

13. C. S. Cundy, J. O. Forrest, and R. J. Plaisted, Micropor. Mesopor. Mater. 66 (2003) 143-156. 